Article

\title{
The Systemic Immune Response in COVID-19 Is Associated with a Shift to Formyl-Peptide Unresponsive Eosinophils
}

\author{
Leo Koenderman 1,2,*, Maarten J. Siemers 1,2, Corneli van Aalst 1,2, Suzanne H. Bongers 2,3, Bas J.J. Bindels 1,2, \\ Giulio Giustarini ${ }^{1,2}$, Harriët M.R. van Goor ${ }^{4}$, Karin A.H. Kaasjager ${ }^{4}$ and Nienke Vrisekoop ${ }^{1,2}$ \\ 1 Department of Respiratory Medicine; m.j.siemers@students.uu.nl (M.J.S.); c.vanaalst@umcutrecht.nl \\ (C.v.A.); b.j.j.bindels@students.uu.nl (B.J.J.B.); G.Giustarini@umcutrecht.nl (G.G.); n.vrisekoop@um- \\ cutrecht.nl (N.V.) \\ 2 Center for Translational Immunology \\ 3 Department of Trauma Surgery; s.h.bongers@umcutrecht.nl \\ 4 Department of Internal Medicine; H.M.R.vanGoor-3@umcutrecht.nl (H.M.R.v.G.); h.a.h.kaasjager@um- \\ cutrecht.nl (K.A.H.K.) \\ * Correspondence: 1.koenderman@umcutrecht.nl; Tel.: +31-88-7556180
}

\begin{abstract}
A malfunction of the innate immune response in COVID-19 is associated with eosinopenia particularly in more severe cases. This study tested the hypothesis that this eosinopenia is COVID19 specific and is associated with systemic activation of eosinophils. Blood of 15 healthy controls and 75 adult patients with suspected COVID-19 at the ER were included before PCR testing and analyzed by point-of-care automated flow cytometry (CD10, CD11b, CD16 and CD62L) in the absence or presence of a formyl peptide (fNLF). 45 SARS-CoV-2 PCR positive patients were grouped based on disease severity. PCR negative patients with proven bacterial $(n=20)$ or other viral $(n=10)$ infections were used as disease controls. Eosinophils were identified with the use of the FlowSom algorithm. Low blood eosinophil numbers $(<100$ cells/ $\mu \mathrm{l} ; \mathrm{p}<0.005)$ were found both in patients with COVID-19 and with other infectious diseases albeit less pronounced. Two discrete eosinophil populations were identified in healthy controls both before and after activation with $\mathrm{fNLF}$ based on the expression of CD11b. Before activation, the CD11b bright population consisted of $5.4 \%(C 195 \%=3.8,13.4)$ of total eosinophils. After activation, this population of CD11b bright cells comprised nearly half the population $\left(42.21 \%, \mathrm{CI}_{95 \%}=35.9,54.1\right)$. Eosinophils in COVID-19 had a similar percentage of CD11b bright cells before activation $\left(7.6 \%, \mathrm{CI}_{95} \%=4.5,13.6\right)$, but were clearly refractory to activation with fNLF as a much lower percentage of cells end up in the CD11b bright fraction after activation $\left(23.7 \%, \mathrm{CI}_{95 \%}=18.5,27.6\right.$; $\left.\mathrm{p}<0.001\right)$. Low eosinophil numbers in COVID-19 are associated with refractoriness in responsiveness to fNLF, which is likely caused by migration of fully functional cells to the tissue. Such homing might be beneficial as eosinophils have been implicated in viral killing.
\end{abstract}

Keywords: SARS-CoV-2; COVID-19; point-of-care flow cytometry; eosinophil; formyl peptide; responsiveness; blood

\section{Introduction}

Coronavirus disease 2019 (COVID-19), a rapidly emerging pandemic disease caused by the Severe Acute Respiratory Syndrome Coronavirus 2 (SARS-CoV-2), causes an extremely variable disease ranging from asymptomatic to viral pneumonia, respiratory distress, ICU-admission and/or death [1,2]. There are still many questions regarding the dominant pathogenetic mechanisms underlying the cause and course of disease [2]. Several hypotheses have been put forward and two of these that are not mutually exclusive have received a lot of attention in the recent literature: coagulopathy associated with tissue edema and (micro)thrombosis [3] and hyperactivation of the innate immune system [4] associated with a cytokine storm [5].

Clinically severe to critical COVID-19 in the ICU has many characteristics of coagulopathy and tissue edema particularly in the lung [6]. This supports the concept that 
ARDS in COVID-19 is mainly caused by tissue edema that is mediated by a deregulated kininogen-bradykinin pathway [3]. Supporting evidence comes from the fact that the SARS-CoV2 virus uses angiotensin-converting enzyme-2 (ACE-2) as porte-d'entrée in epithelial cells, which is associated with downregulation of ACE-2 from the cell surface [2]. A decrease in ACE-2 is thought to be involved in the attenuated breakdown of bradykinin [3] leading to tissue edema in the lung and a clinical picture reminiscent of ARDS [6]. Despite its significance, coagulopathy is beyond the scope of this article which is focused on a putative dysfunction of the innate immune response [4].

A multitude of articles describe the importance of hyperinflammation in the pathogenesis of COVID-19 (see for recent reviews [2,5,7]). Most of these studies had a case control design using healthy individuals as controls. These studies generally show more systemic inflammation with increasing disease severity. This is associated with a cytokine storm and clear differences in the phenotype and/or activation status of the different innate immune cells [8]. Deep phenotyping has identified several neutrophil phenotypes leading to the conclusion that hyperinflammation is a specific and integral part of the pathogenesis of COVID-19. However, recent studies refute the importance of a cytokine storm and neutrophil activation in the pathogenesis of COVID-19 at least in the population of patients outside the intensive care unit. In fact, these studies imply that these mechanisms are commonly initiated in any severe/critical inflammatory disease and are not COVID-19 specific [1,9].

The importance of the eosinophilic compartment in the pathogenesis of COVID-19 is less clear. Early studies particularly those coming from Wuhan have indicated that more severe COVID-19 is associated with eosinopenia [10,11]. Unfortunately, no underlying mechanism was identified. Also the activation status of the remaining eosinophils in the peripheral blood was not studied.

Here we set out experiments to study the eosinophil compartment in COVID-19 patients both qualitatively and quantitatively. We will show that also our COVID-19 cohort was associated with a relative eosinopenia, but eosinopenia was also seen in some patients with other viral and bacterial infections albeit to a lower extent. In addition, the eosinophil compartment exhibited clear signs of deregulated activation. Whether this activation is pathological or a normal response to virus infections will be discussed.

\section{Materials and Methods}

\subsection{Study Design}

Seventy-five suspected COVID-19 patients older than 18 years of age were included who presented in the University Medical Center Utrecht (UMCU) during the Dutch epidemic between March 19th 2020 and May 17th 2020. SARS-CoV2 PCR positive patients $(n=45)$ were compared to healthy controls $(n=15)$ and SARS-CoV2 PCR negative patients with an alternative diagnosis (viral $(n=10)$ or bacterial infection $(n=20)$ ). The initial blood samples were drawn at the emergency room (ER) or within two days on the COVID-19 ward. Hereafter, samples were drawn at regular intervals during stay at the COVID-19 ward. No blood samples were drawn at the intensive care unit. Clinical outcomes and baseline characteristics of the COVID-19 patients have been published recently [9]. The patients were not immunocompromised nor used immunosuppressive medication according to the International Classification of Disease, 9th revision [12].

\subsection{Study Procedure}

Blood was drawn in one extra $4 \mathrm{~mL}$ or $9 \mathrm{~mL}$ VACUETTE ${ }^{\circledR}$ sodium heparin blood tube (Greiner Bio-One, Kremsmünster, Austria) from each patient during standard-of-care diagnostic workup at the Emergency Department or during standard blood drawing moments at the COVID-19 ward. Healthy control blood was obtained from the Mini Donor Service at the UMCU. Healthy control subjects could not be matched for age nor gender because of the limitations for clinical experiments during the first wave of the pandemic and had to be chosen based on donor availability. The blood from healthy controls was 
drawn at the out-patient clinic, which is next to the emergency department. Sample handling times analysis were identical to the patients included in this study.

\subsection{Flow Cytometry Analysis by the Automated AQUIOS CL ${ }^{\circledR}$ "Load E Go" Flow Cytometer}

Blood was analyzed in terms of total cell counts, scatter and fluorescence by a completely automated point-of-care AQUIOS CL ${ }^{\circledR}$ "Load \& Go" flow cytometer (Beckman Coulter, Miami, FL, USA) exactly as described before [13]. Aspecific granulocyte activation in the blood tube ex vivo was minimized by a applying a short $(<60 \mathrm{~min})$ time between blood drawing and analysis [13]. The analysis of the blood samples was exactly as described before [13]. In short, the whole blood samples were stained by a customized antibody mix (CD16-FITC (clone 3G8, Beckman Coulter, Miami, FL, USA), CD11b-PE (clone Bear1, Beckman Coulter), CD62L-ECD (clone DREG56, Beckman Coulter), CD10PC5 (clone ALB1, Beckman Coulter) and CD64-PC7 (clone 22, Beckman Coulter) in the absence (control) and presence (activation) of the bacterial/mitochondrial-derived stimulus N-formyl-norleucyl-leucyl-phenylalanine (10 $\mu \mathrm{M}$ fNLF) (BioCat GmbH, Heidelberg, Germany). Hereafter, the red blood cells were lysed and the data were analyzed and exported as .lmd files for later in depth analysis. Aspecific granulocyte activation in the blood tube ex vivo was minimized by a applying a short $(<60 \mathrm{~min})$ time between blood drawing and analysis [13].

\section{A: Granulocyte gate}

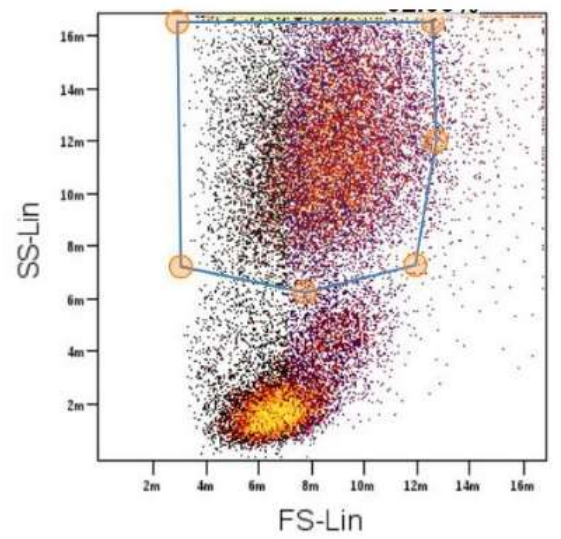

\section{B: Non-Neutrophils gate}

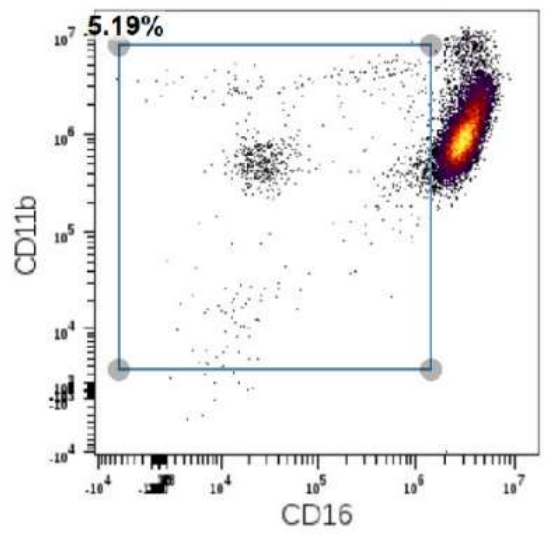

\section{C: Metacluster containing eosinophils}

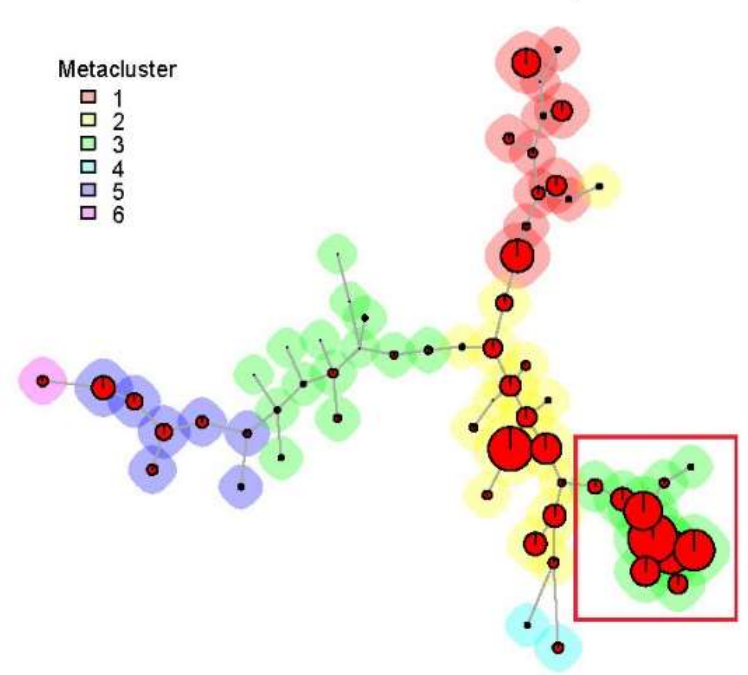




\section{D: 2D visualisation of the eosinophil metacluster: $\mathrm{CD} 16^{-} / \mathrm{SSC}^{\mathrm{High}}$ cells}

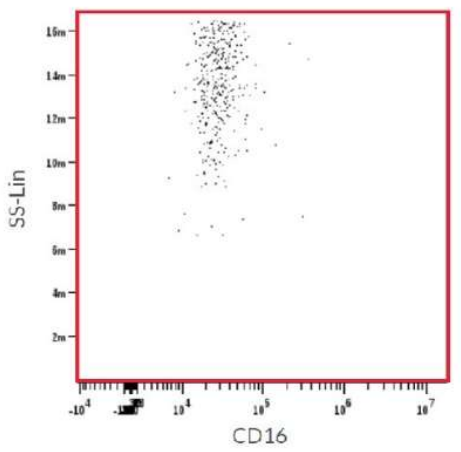

\section{E: 2D visualisation of the eosinophil metacluster: CD16/CD11b moderate cells}

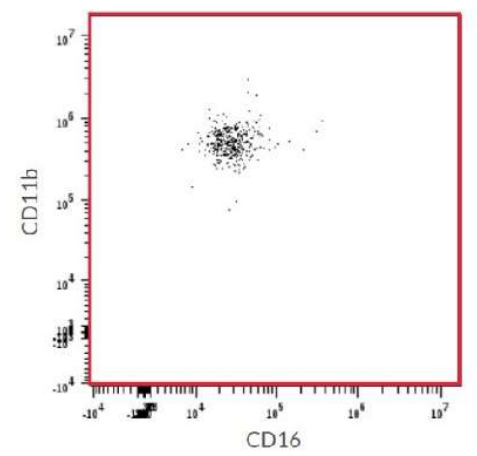

Figure 1. Gating strategy and identification of the eosinophil compartment. Panel A/B: Gating strategy for obtaining cells in the granulocyte gate (A) that are not neutrophils (B). Flow cytometric analysis was done using the online tool Cytobank (www.cytobank.org). Panel C. Identification of eosinophils (metacluster 3 present in the square) with the use of the FlowSom algorithm ([14] present in Cytobank). Panels D/E: characterization of cells in metacluster 3 as being SSC high and CD16 negative (D) and CD11b moderate (E) eosinophils.

\subsection{Analysis of Flow Cytometry Data}

The .lmd data files obtained by AQUIOS CL ${ }^{\circledR}$ were imported into the online tool Cytobank (www.cytobank.org, Santa Clara, CA). The identification of eosinophils in the blood samples was done by gating first on granulocytes on the basis of scatter characteristics (see Figure 1). Hereafter, a second gate was set outside the majority of neutrophils (CD16 $\left.{ }^{\text {bright}) ~ t o ~ e n r i c h ~ f o r ~ e o s i n o p h i l s ~(C D 16 ~}{ }^{\text {negative }}\right)$ and progenitors (CD16 $\left.{ }^{\text {dim }}\right)$. Hereafter, this fraction was analyzed by the FlowSom algorithm [14] (present in Cytobank) choosing 64 nodes and 6 metaclusters (figure 1C). Next, the data were analyzed to establish that metacluster three contained the eosinophils (SSChigh CD16 ${ }^{\text {negative }}$ and CD11b $b^{\text {moderate }}$ ) (Figure $1 \mathrm{D}$ and $1 \mathrm{E})$. In this metacluster the percentage and the fNLF responsiveness of the eosinophils present in the sample was determined. To this end, the eosinophil markers were analyzed in the absence (resting) and presence (activated) of fNLF (10 $\mu \mathrm{M})$.

Two eosinophil phenotypes could be discriminated by either moderate or high expression of CD11b (see figure 2). The marker CD64 was used to facilitate differentiation between viral and bacterial infections in COVID-19 negative patients, as has been demonstrated before [15]. CD64 was not applied for uni -and bivariate analysis of the eosinophil compartment.

\subsection{Clinical Characteristics of Suspected COVID-19}

Patients that tested PCR positive for SARS-CoV-2 at any point during admission were considered having COVID-19. Patients that were PCR negative for COVID-19 were studied in detail for other diagnoses explaining their clinical condition applying bacterial cultures, PCR's for other viruses, or other diagnostic tests. PCR negative patients with no other explaining diagnosis were excluded from the study.

Patients that were PCR positive for SARS-CoV-2 were grouped based on disease severity according WHO criteria published on May 27th 2020 (Clinical Management of COVID-19 [16]). Moderate disease was defined by the presence of mild to moderate pneumonia (including SpO2 $\geq 90 \%$ on room air). The definition of severe disease included severe pneumonia (including SpO2 $<90 \%$ on room air) and one of the following: respiratory rate $>30$ breaths/min; severe respiratory distress, but without meeting the Berlin criteria [17] for ARDS at any time during admission. Critical disease was defined by the presence of $\mathrm{ARDS} \mathrm{SpO}_{2} / \mathrm{FiO}_{2} \leq 315$ [16] at any point during admission. 


\subsection{Study Approval}

For this study, a waiver for formal ethical approval was provided by the institutional medical ethics committee under protocol number 20-284/C. The Mini Donor Service received positive approval from the medical ethical committee of the UMCU under protocol number 07-125/C. All procedures performed in this study were in accordance with the 1964 Helsinki declaration and its later amendments.

\subsection{Statistics}

Kruskal-Wallis analysis was applied for the eosinophil data when multiple groups were compared. For comparison of CD11b bright and CD11b low cells a Wilcoxon matchedpairs signed rank test was used. Healthy individuals and COVID-19 PCR negative patients with proven bacterial or other viral infection were used as separate control groups. Differences in receptor expression on eosinophils were tested with Kruskal-Wallis, and post-hoc, the Dunn's test with Bonferonni correction was used for multiple comparison. GraphPad Prism version 7 (GraphPad software inc., San Diego, CA, USA) was used for analysis and data visualization. Statistical significance was defined as a p-value $<0.05$.

\section{Results}

3.1. Responsiveness for fNLF Identifies Two Eosinophil Phenotypes in the Peripheral Blood of Healthy Control Individuals

Automated flowcytometry allows quick (25 min post venipuncture) determination of the phenotype of leukocytes with a minimal artificial activation due to ex vivo manipulation of the cells [13]. In fact, we have shown that expression of activation markers such as Mac-1/CD11b on eosinophils is quickly induced in the blood sample in the collection tube without any additional manipulation. This artificial activation is for a large part prevented by the fast analysis in the AQUIOS [13]. Applying this method we identified two discrete populations of eosinphils in healthy control individuals: a dominant population that is characterized by a low expression of CD11b (CD11b boderate) and a much smaller population of CD11b bright eosinophils (Figure 2).

\section{A: Expression of CD11b on eosinophils with and without fNLF}
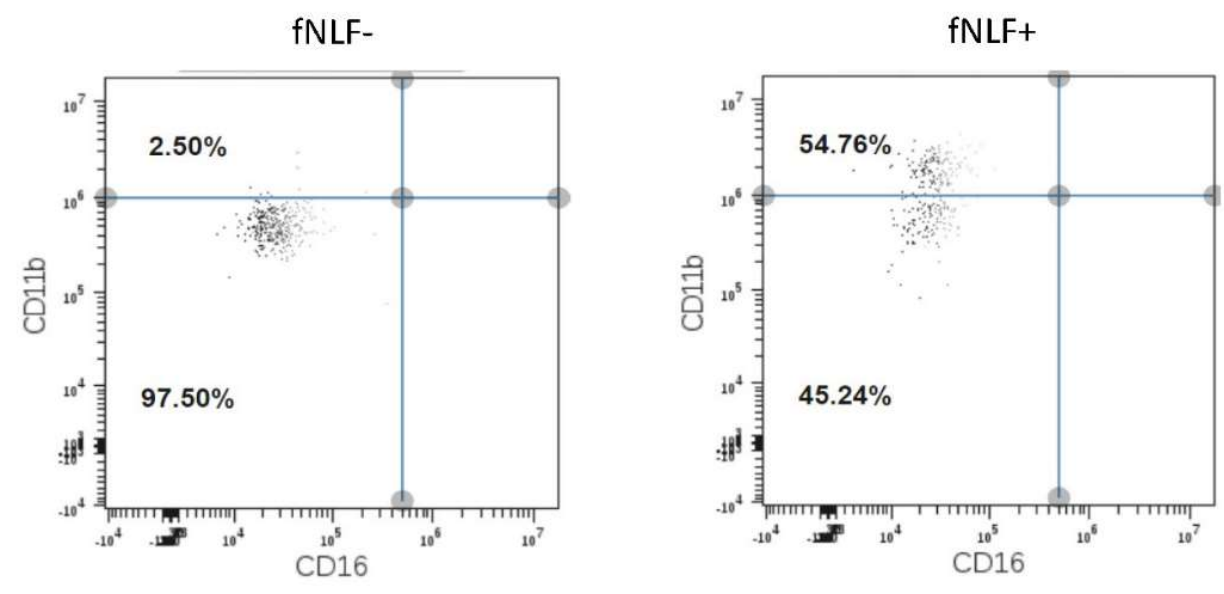


\section{B: Percentage CD11b ${ }^{\text {bright }}$ cells}

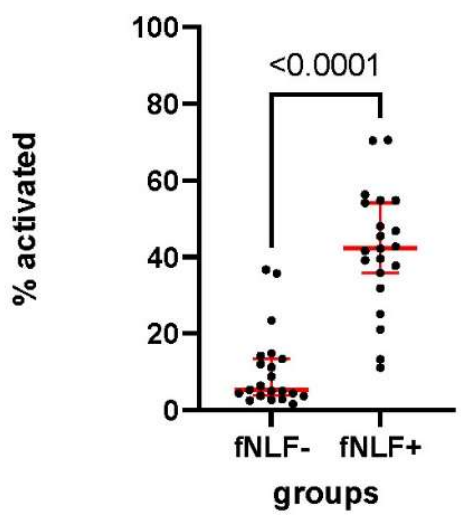

2C: Expression of CD62L on CD11b $\mathrm{b}^{\text {Bright }}$ and CD11b ${ }^{\text {moderate }}$ eosinophils

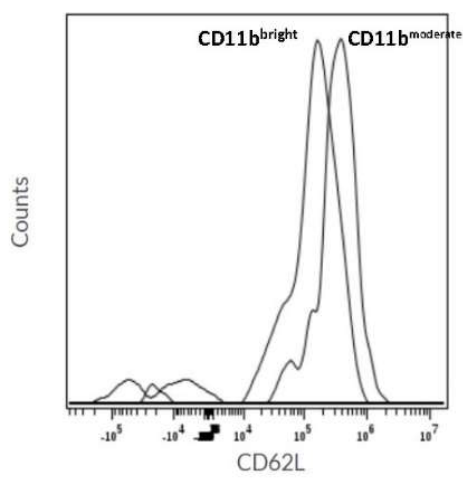

Figure 2. Identification of two types of eosinophils characterized by differences in responsiveness for formyl peptides. Panel A: expression of CD11b on eosinophils from normal healthy controls in the absence of the formyl-peptide fNLF. Expression of CD11b (panel B) in the presence of fNLF (10 $\mu \mathrm{M})$. Data are expressed as percentage CD11bright cells present in upper left quadrant. CD11 $b^{\text {moder- }}$ ate cells are characterized by a high expression of CD62L (panel C). Data in panel B are analysed using a Wilcoxon matched-pairs signed rank test.

Eosinophils have been described to be responsive to the formyl-peptide formyl-methionyl-leucyl phenylalanin (fMLF [18]) and this response is sensitive for priming by inflammatory mediators [19]. Unfortunately, the methionyl moiety in fMLF is sensitive for oxidation that leads to the biological inactivity of the peptide. This precludes application in our automate flow analysis as the formyl-peptide is present in the deep well plate as long as required for all the analyses. In marked contrast to fMLF that looses its activity in days fNLF is stable for weeks to months [9]. Activation of whole blood with fNLF (10 $\mu \mathrm{M})$ leads to a marked increase in cells found in the CD11bright population (Figure 2). Interestingly, a sizeble number of eosinophils in the CD11b boderate did not respond to fNLF (Figure 2). In line, CD62L was shed in the CD11b bright but not in the CD11b ${ }^{\text {moderate }}$ population (Figure $2 \mathrm{C}$ ). The small number of eosinophils with the CD11b bright phenotype did not further upregulate CD11b in response to fNLF (Figure 2). 
3.2. Eosinophil Numbers in the Peripheral Blood of COVID-19 Patients, Disease Controls and Healthy Controls.

\section{A: Absolute count of eosinophils at admission}

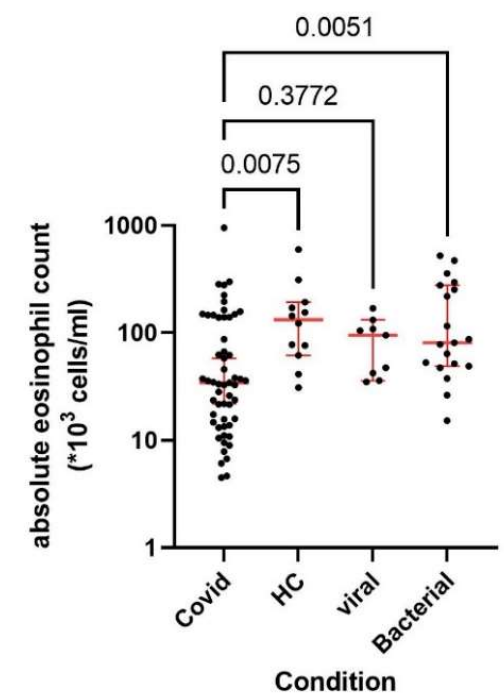

\section{B: Absolute count of eosinophils over time}

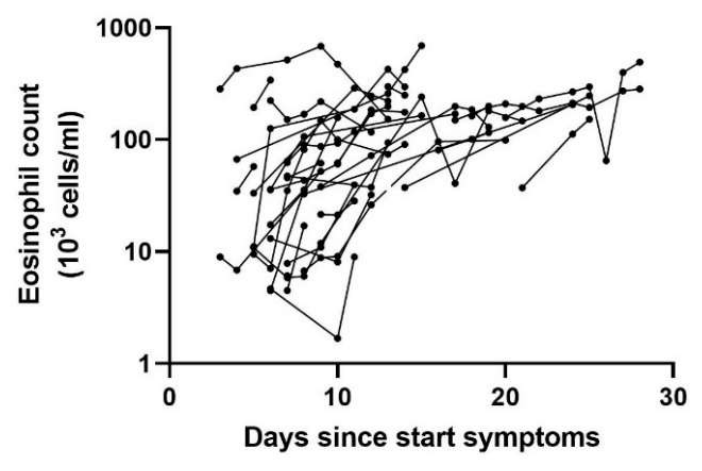

Figure 3. Eosinophil counts under healthy and infectious conditions. A. The absolute number of eosinophils in the blood of different patient groups and controls at admission. Data are expressed as medians +/- confidence interval and statistical significance was tested by Kruskal Wallis test with Dunns test for multiple comparison between groups. B. Blood of COVID-19 patients was drawn at different times after admission to the hospital. The data points of individual are connected.

Figure 3 shows the absolute eosinophil counts in the peripheral blood of the different categories of patients and controls at presentation in the hospital (Panel A, see also [9]). Eosinophils were identified on the basis of metacluster 3 identified by the FlowSom algorithm [14] (see Figure 1 and the M\&M section). In some experiments this gate was validated by additional experiments using the eosinophil marker CCR3 (CD193) (results not shown). As shown before [9], COVID-19 patients were characterized by a relative eosinopenia but such eosinopenia was also found in the patients with other infections albeit less pronounced ( $p=0.005$, Figure $3 \mathrm{~A}$ ). The numbers of blood eosinophils were not correlated with disease severity (not shown). The eosinophil numbers normalized with after infection (see Figure 3B). 


\section{A: Percentage CD11b $\mathrm{b}^{\text {bright }}$ eosinophils}

fNLF-

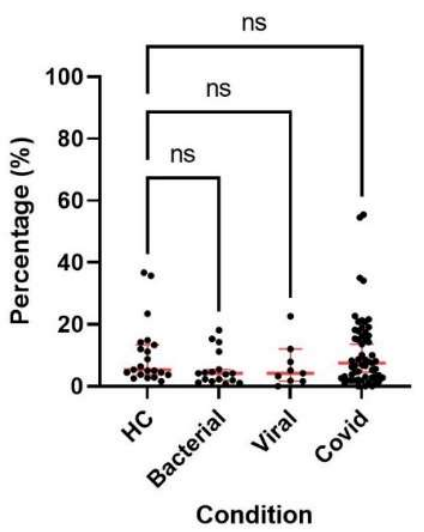

C: Percentage CD11b bright eosinophils in COVID-19

fNLF-

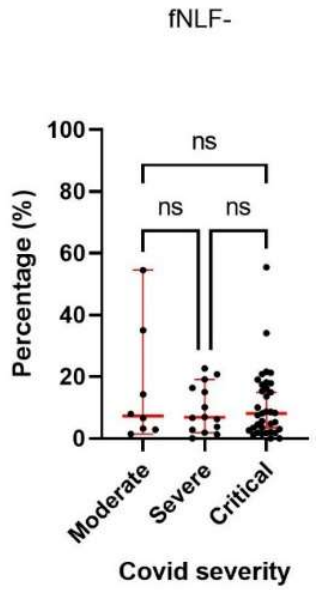

B: Percentage CD11b $\mathrm{b}^{\text {bright }}$ eosinophils

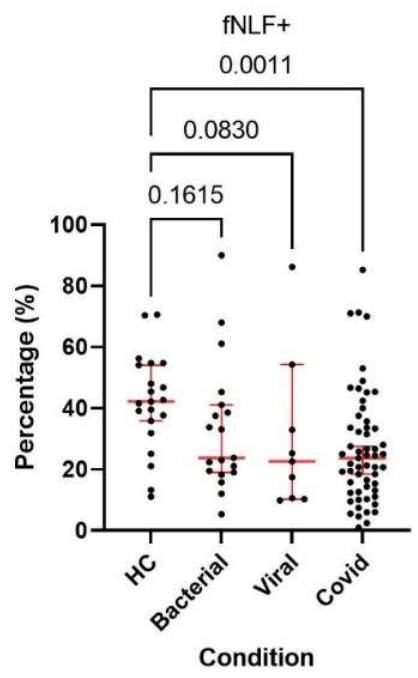

\section{eosinophils in COVID-19}

fNLF+

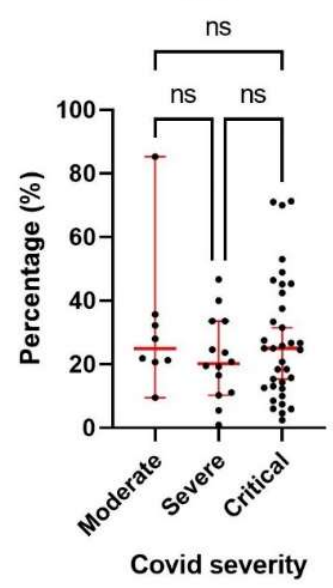

Figure 4. Responsiveness of the eosinophil compartment for formylpeptides in patients with different types of infection. The percentage CD11b brigth eosinophils in the blood of different patient groups and controls at admission in the absence (panel A) or presence (panel B) of the formyl peptide fNLF $(10 \mu \mathrm{M})$. The percentage of CD11b bright eosinophils in the blood of COVID-19 patients in the absence (panel C) and presence (panel D) of fNLF (10 mM). Data are expressed as medians +/confidence interval and statistical significance is tested by Kruskal Wallis test with Dunns test for multiple comparison between groups.

3.3. Responsiveness of the Eosinophil Phenotypes for fNLF at Presentation in the Hospital: Comparison between COVID-19 Patients, Disease Controls and Healthy Controls.

The responsiveness for fNLF was determined for eosinophils from the blood of COVID-19 patients and the different control groups such as described above. In marked contrast to healthy controls, eosinophils in the blood of COVID-19 patients were refractory 
to activation with fNLF (see Figure 4). Interestingly, this refractoriness is not specific for COVID-19 as both bacterial and viral infections lead to a similar refractoriness of responsiveness to fNLF.

\subsection{Normalization of Eosinophil counts and Responsiveness to fNLF during Resolving Disease in the COVID-19 Ward.}

Next we tested whether eosinophil counts and eosinophil responsiveness for fNLF would normalize during resolving disease. Figure 5 shows that during the disease course eosinophil numbers increased and the refractoriness for formyl-peptides decreased during amelioration of disease. The data are consistent with the hypothesis that the time needed for recovery mirrors the time of normalization of the eosinophils compartment.
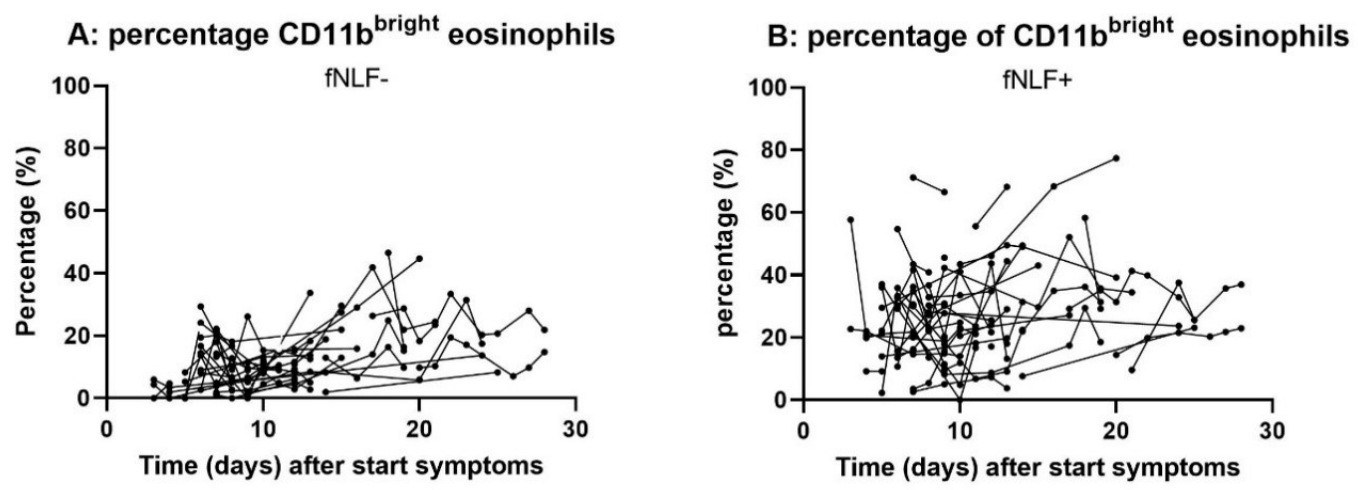

Figure 5. Percentage of CD11b bright eosinophils in the peripheral blood of COVID-19 patients during resolution of the disease. Blood of COVID-19 patients was drawn at different times after admission to the hospital in the absence (panel A) or presence (panel B) of fNLF $(10 \mu \mathrm{M})$. The data points of individual are connected.

\section{Discussion}

The fast and automated point-of-care flow analysis allowed the definition of two separate eosinophil subsets in healthy controls: cells that are responsive to fNLF and those that are unresponsive. fNLF unresponsive cells are CD11b boderate and CD62 Lright, and do not shift to CD11b bright cells upon activation with fNLF (see Figure 1). The discrete CD11b bright population likely consists of activated cells as part of the eosinophils in the CD11b boderate population move to the CD11b bright population after activation of whole blood with fNLF in vitro. It is, therefore, tempting to speculate that the fNLF unresponsive cells belong to a separate subset of eosinophils. Such hypothesis is supported by the data published by Mesnil and colleagues suggesting separate subsets of resident and inflammatory eosinophils [20]. The resident subset was characterized by a high expression of Lselectin (CD62L). Unresponsive CD11b moderate eosinophils might belong to this resident subset as our data show that this population is characterized by a high CD62L expression as well (see Figure 2C). However, future studies should focus on this hypothesis.

We next applied our methodology for eosinophil phenotyping to patients with COVID-19 as eosinophils have been implicated in the pathogenesis of the disease. This is mainly based on the finding that eosinopenia is a hall-mark of the disease and the suggestion that the extend of eosinopenia is correlated with the severity of disease [10,11]. Our data support the low eosinophil counts in COVID-19 patients at presentation in the hospital, but contrasts the suggestion that this eosinopenia is specific for infection with SARSCoV-2 [10,11]. This conclusion is based on the fact that some of the included COVID-19 PCR negative patients suffering from either bacterial or (non-COVID-19) viral infection were also characterized by a low number of eosinophils albeit less pronounced. Apparently, acute systemic inflammation evoked by infection can lead to eosinopenia irrespec- 
tive of the micro-organism. This conclusion is strengthened by our previous study showing that challenge of healthy volunteers with the bacterial microbe associated molecular pattern (MAMP), lipopolysaccharide (LPS) leads to acute transient eosinopenia [21]. Together these findings show the surprising result that the eosinophil, historically seen as a typical T2-effector cells, responds to a non-T2 general MAMP in vivo. This supports the view that eosinophils in vivo can be responsive to a non-T2 inflammatory cue.

The eosinophil compartment in the blood of COVID-19 patients is associated with refractoriness for activation with fNLF. This impaired responsiveness is associated with low eosinophil counts. It is. Therefore, tempting to speculate that the responsive cells leave the blood and migrate to tissues, leaving behind the refractory cells. This is in line with the idea that inflammatory eosinophils are highly responsive to inflammatory and infectious cues, whereas refractory eosinophils are not. This latter subset might home for the tissues that contain eosinophils in homeostasis [22]. The concept of disappearance of responsive eosinophils from the blood during disease has been put forward in several studies focusing on pathogenesis of allergic asthma [23,24]. This leads to a counterintuitive concept that activation of the eosinophil compartment is associated with the presence of non-responsive cells in the peripheral blood. Support for this type of reasoning is the finding that responsive eosinophil re-emerge in the peripheral blood during resolution of disease (see Figure 5). Importantly, the increase in refractory eosinophils in peripheral blood is not specific for COVID-19 as similar findings were obtained in SARS-CoV-2 PCR negative patients suffering from other infections.

Responsiveness of eosinophils for fNLF was anticipated as we have described fMLFinduced activation in vitro $[18,19]$. However, in those old experiments the magnitude of activation varied considerably between donors. As these data were obtained in cell suspensions with no single cell information low activation could be interpreted two ways: low responsiveness of all eosinophils, or a low number of highly responsive cells with the majority being non-responsive. Our data support the latter hypothesis. This data also shows that at least part of the eosinophil compartment in humans is responsive to bacterial MAMP's such as fNLF and another part is not. This implies that eosinophils, historically seen as T2-effectors cells involved allergic and parasitic diseases, are likely to engage in bacterial infections in humans in vivo as well. This latter hypothesis is strengthened by earlier studies showing the capability of eosinophils to kill bacteria [25] and viruses [26].

These data lead to the essential question whether homing of responsive eosinophils to the tissues in COVID-19 should be prevented? Obviously, this descriptive study will not lead to an answer, but several important issues should be taken into account. Firstly, eosinophils have been shown to kill viruses in vitro through the presence of RNAses present in the granules of these cells [26]. Secondly, we have recently shown that eosinophils in asthma patients have lost their capacity to kill viruses and this is associated with more disease [27]. On the other hand, inflammatory eosinophils present in allergic inflamed tissue have been associated with collateral damage to the host tissues by liberation of granule proteins such as major basic protein (MBP [28]). The pathogenetic role of eosinophils in at least allergic disease is supported by the finding that inhaled corticosteroids are the key therapy in allergic asthma and is associated with clear suppression of tissue eosinophilia [29]. Therefore, it is still an open issue under which disease conditions eosinophils are guilty by association, play a beneficial role or play a part in the pathogenesis of the disease. Only the latter situation warrants an intervention targeting these cells.

In summary, the issue whether a specific hyperinflammation in the pathogenesis of COVID-19 is the cause or rather the consequence of infection with the SARS-CoV2 is still a matter of debate. At presentation, COVID-19 patients in our cohort show only very limited signs of a specific systemic inflammation and they cannot be discriminated from patients infected with other micro-organisms or viruses. The initiation of disease can still be caused by aberrations in the bradykinin metabolism [3] and coagulopathy [30]. The resulting lung edema and thrombo-embolic events [31] can lead to ischemia and reperfusion injury leading to tissue damage and release of damage associated molecular patterns (DAMP's) including mitochondrial derived formyl-peptides. This can then lead to a toxic 
triangle of edema, embolisms and inflammation. In this scenario inflammation is more the consequence rather than the cause of the disease. The role of eosinophils in the pathogenesis of COVID-19 is uncertain, but targeting these cells is only opportune when it is clear that these cells are not beneficial as virus killing cells.

Author Contributions: Conceptualization, L.K., H.G., K.K. and N.V.; methodology, M.S., B.B., G.G, and H.G; software, M.S. and B.B.; validation, L.K. M.S. and N.V.; formal analysis, L.K. and M.S.; investigation C.V. and S.B.; resources L.K.; data curation, B.B; writing-original draft preparation, L.K. writing - review and editing, ALL.; visualization, M.S.; supervision, X.X.; project administration, L.K., M.S., and N.V.; funding acquisition, L.K. All authors have read and agreed to the published version of the manuscript.

Funding: This research was funded by grants (grant \# 400.17.604) of the Dutch Research Council (NWO) in the framework of the "Startimpulse" Dutch National Research Agenda (NWA) and by Health Holland (grant \# 20064).

Acknowledgments: For technical support we want to thank Paul van Hoof, Roelof-Jan van der Lei, Geert Weijers, Andreas Boehmler and Markus Kaymer from the Beckman Coulter team.

Conflicts of Interest: The AQUIOS CL ${ }^{\circledR}$ "load \& go" flow cytometer is provided by the company Beckman Coulter Life Sciences, Miami, FL, USA. All authors declare that there are no other competing interests.

\section{References}

1. Leisman DE, Ronner L, Pinotti R, Taylor MD, Sinha P, Calfee CS, Hirayama AV, Mastroiani F, Turtle CJ, Harhay MO, Legrand M, Deutschman CS. Cytokine elevation in severe and critical COVID-19: a rapid systematic review, meta-analysis, and comparison with other inflammatory syndromes. Lancet Respir Med. 2020 Dec;8(12):1233-1244. doi: 10.1016/S22132600(20)30404-5. Epub 2020 Oct 16. PMID: 33075298; PMCID: PMC7567529.

2. Wiersinga WJ, Rhodes A, Cheng AC, Peacock SJ, Prescott HC. Pathophysiology, Transmission, Diagnosis, and Treatment of Coronavirus Disease 2019 (COVID-19): A Review. JAMA. 2020 Aug 25;324(8):782-793. doi: 10.1001/jama.2020.12839. PMID: 32648899.

3. van de Veerdonk FL, Netea MG, van Deuren M, van der Meer JW, de Mast Q, Brüggemann RJ, van der Hoeven H. Kallikrein-kinin blockade in patients with COVID-19 to prevent acute respiratory distress syndrome. Elife. 2020 Apr 27;9:e57555. doi: 10.7554/eLife.57555. PMID: 32338605; PMCID: PMC7213974.

4. Vardhana SA, Wolchok JD. The many faces of the anti-COVID immune response. J Exp Med. 2020 Jun 1;217(6):e20200678. doi: 10.1084/jem.20200678. PMID: 32353870; PMCID: PMC7191310.

5. Wang J, Jiang M, Chen X, Montaner LJ. Cytokine storm and leukocyte changes in mild versus severe SARS-CoV-2 infection: Review of 3939 COVID-19 patients in China and emerging pathogenesis and therapy concepts. J Leukoc Biol. 2020 Jul;108(1):17-41. doi: 10.1002/JLB.3COVR0520-272R. Epub 2020 Jun 13. PMID: 32534467; PMCID: PMC7323250.

6. Li X, Ma X. Acute respiratory failure in COVID-19: is it "typical" ARDS? Crit Care. 2020 May 6;24(1):198. doi: 10.1186/s13054020-02911-9. PMID: 32375845; PMCID: PMC7202792.

7. Gustine JN, Jones D. Immunopathology of Hyperinflammation in COVID-19. Am J Pathol. 2021 Jan;191(1):4-17. doi: 10.1016/j.ajpath.2020.08.009. Epub 2020 Sep 11. PMID: 32919977; PMCID: PMC7484812.

8. Spijkerman R, Bongers SH, Bindels BJJ, Tinnevelt GH, Giustarini G, Jorritsma NKN, Buitenwerf W, van Spengler DEJ, Delemarre EM Nierkens, van Goor HMR, Jansen JJ, Vrisekoop N, Hietbrink F, Leenen LPH, Kaasjager KAH, Koenderman L. Flow cytometric evaluation of the neutrophil compartment in COVID-19 at hospital presentation: A normal response to an abnormal situation. J. Leukoc. Biol. 2021; 109(1):99-114. DOI: 10.1002/JLB.5COVA0820-520RRR

9. Zhang JJ, Dong X, Cao YY, Yuan YD, Yang YB, Yan YQ, Akdis CA, Gao YD. Clinical characteristics of 140 patients infected with SARS-CoV-2 in Wuhan, China. Allergy. 2020 Jul;75(7):1730-1741. doi: 10.1111/all.14238. Epub 2020 Feb 27. PMID: 32077115.

10. Roca E, Ventura L, Zattra CM, Lombardi C. EOSINOPENIA: An early, effective, and relevant COVID-19 biomarker? QJM. 2020 Sep 2:hcaa259. doi: 10.1093/qjmed/hcaa259. Epub ahead of print. PMID: 32877511; PMCID: PMC7499754.

11. Guan, W., Ni, Z., Hu, Y., et al. Clinical characteristics of coronavirus disease 2019 in China. N Engl J Med . Epub ahead of print 2020. DOI: 10.1056/NEJMoa2002032.

12. World Health Organisation (WHO). International Statistical Classification of Diseases and Related Health Problems (International Classification of Diseases)(ICD) 10th Revision - Version:2010. 2010.

13. Spijkerman, R., Hesselink, L., Hellebrekers, .P, et al. Automated flow cytometry enables high performance point-of-care analysis of leukocyte phenotypes. J Immunol Methods. 2019;474:112646.

14. Van Gassen S, Callebaut B, Van Helden MJ, Lambrecht BN, Demeester P, Dhaene T, Saeys Y. FlowSOM: Using self-organizing maps for visualization and interpretation of cytometry data. Cytometry A. 2015 Jul;87(7):636-45. doi: 10.1002/cyto.a.22625. Epub 2015 Jan 8. PMID: 25573116.

15. Hoffmann JJ. Neutrophil CD64: a diagnostic marker for infection and sepsis. Clin Chem Lab Med. 2009;47(8):903-16. doi: 
10.1515/CCLM.2009.224. PMID: 19642859.

16. World Health Organization. (2020). Clinical management of severe acute respiratory infection when novel coronavirus (2019-nCoV) infection is suspected: interim guidance, 28 January 2020. World Health Organization. https://apps.who.int/iris/handle/10665/330893

17. The ARDS Definition Task Force. Acute Respiratory Distress Syndrome. JAMA. 2012;307:2526-2533.

18. Yazdanbakhsh M, Eckmann CM, Koenderman L, Verhoeven AJ, Roos D. Eosinophils do respond to fMLP. Blood. 1987 Aug;70(2):379-83. PMID: 3607277.

19. Koenderman L, Kok PT, Hamelink ML, Verhoeven AJ, Bruijnzeel PL. An improved method for the isolation of eosinophilic granulocytes from peripheral blood of normal individuals. J Leukoc Biol. 1988 Aug;44(2):79-86. doi: 10.1002/jlb.44.2.79. PMID: 3404069.

20. Mesnil C, Raulier S, Paulissen G, Xiao X, Birrell MA, Pirottin D, Janss T, Starkl P, Ramery E, Henket M, Schleich FN, Radermecker M, Thielemans K, Gillet L, Thiry M, Belvisi MG, Louis R, Desmet C, Marichal T, Bureau F. Lung-resident eosinophils represent a distinct regulatory eosinophil subset. J Clin Invest. 2016 Sep 1;126(9):3279-95. doi: 10.1172/JCI85664. Epub 2016 Aug 22. PMID: 27548519; PMCID: PMC5004964.

21. Hassani M, Leijte G, Bruse N, Kox M, Pickkers P, Vrisekoop N, Koenderman L. Differentiation and activation of eosinophils in the human bone marrow during experimental human endotoxemia. J Leukoc Biol. 2020 Nov;108(5):1665-1671. doi: 10.1002/JLB.1AB1219-493R. Epub 2020 Jan 10. PMID: 31922294.

22. Marichal T, Mesnil C, Bureau F. Homeostatic Eosinophils: Characteristics and Functions. Front Med (Lausanne). 2017 Jul 11;4:101. doi: 10.3389/fmed.2017.00101. PMID: 28744457; PMCID: PMC5504169.

23. Johansson MW. Eosinophil Activation Status in Separate Compartments and Association with Asthma. Front Med (Lausanne). 2017 Jun 12;4:75. doi: 10.3389/fmed.2017.00075. PMID: 28660189; PMCID: PMC5466952.

24. Luijk B, Lindemans CA, Kanters D, van der Heijde R, Bertics P, Lammers JW, Bates ME, Koenderman L. Gradual increase in priming of human eosinophils during extravasation from peripheral blood to the airways in response to allergen challenge. J Allergy Clin Immunol. 2005 May;115(5):997-1003. doi: 10.1016/j.jaci.2005.02.002. PMID: 15867857.

25. Yazdanbakhsh M, Eckmann CM, Bot AA, Roos D. Bactericidal action of eosinophils from normal human blood. Infect Immun. 1986 Jul;53(1):192-8. doi: 10.1128/IAI.53.1.192-198.1986. PMID: 3522428; PMCID: PMC260096.

26. Rosenberg HF, Domachowske JB. Eosinophils, eosinophil ribonucleases, and their role in host defense against respiratory virus pathogens. J Leukoc Biol. 2001 Nov;70(5):691-8. PMID: 11698487.

27. Sabogal Piñeros YS, Bal SM, Dijkhuis A, Majoor CJ, Dierdorp BS, Dekker T, Hoefsmit EP, Bonta PI, Picavet D, van der Wel NN, Koenderman L, Sterk PJ, Ravanetti L, Lutter R. Eosinophils capture viruses, a capacity that is defective in asthma. Allergy. 2019 Oct;74(10):1898-1909. doi: 10.1111/all.13802. Epub 2019 May 15. PMID: 30934128; PMCID: PMC6852198.

28. Frigas E, Gleich GJ. The eosinophil and the pathophysiology of asthma. J Allergy Clin Immunol. 1986 Apr;77(4):527-37. doi: 10.1016/0091-6749(86)90341-6. PMID: 3514730.

29. Cowan DC, Cowan JO, Palmay R, Williamson A, Taylor DR. Effects of steroid therapy on inflammatory cell subtypes in asthma. Thorax. 2010 May;65(5):384-90. doi: 10.1136/thx.2009.126722. Epub 2009 Dec 8. PMID: 19996343.

30. Iba T, Levy JH, Levi M, Thachil J. Coagulopathy in COVID-19. J Thromb Haemost. 2020 Sep;18(9):2103-2109. doi: 10.1111/jth.14975. Epub 2020 Jul 21. PMID: 32558075; PMCID: PMC7323352.

31. Poissy J, Goutay J, Caplan M, Parmentier E, Duburcq T, Lassalle F, Jeanpierre E, Rauch A, Labreuche J, Susen S; Lille ICU Haemostasis COVID-19 Group. Pulmonary Embolism in Patients With COVID-19: Awareness of an Increased Prevalence. Circulation. 2020 Jul 14;142(2):184-186. doi: 10.1161/CIRCULATIONAHA.120.047430. Epub 2020 Apr 24. PMID: 32330083. 